\title{
Cambios electrocardiográficos asociados a hipopotasemia: Alteraciones del segmento ST imitando isquemia miocárdica de múltiples territorios
}

\author{
Lucrecia María Burgos $^{1}$, María Domínguez ${ }^{2}$, Luciano Battioni ${ }^{3}$, Juan Pablo Costabef ${ }^{4}$, Marcelo Trivi ${ }^{5}$. \\ 1. Residente de cardiología clínica del Instituto Cardiovascular de Buenos Aires (ICBA) \\ 2. Residente de cardiología clínica del Instituto Cardiovascular de Buenos Aires (ICBA) \\ 3. Jefe de residentes de cardiología clínica del Instituto Cardiovascular de Buenos Aires (ICBA) \\ 4. Jefe de Servicio de emergencias del Instituto Cardiovascular de Buenos Aires (ICBA) \\ 5. Jefe de Servicio de cardiología clínica del Instituto Cardiovascular de Buenos Aires (ICBA) \\ No se declaran Conflictos de interés
}

Se presenta el caso de un paciente de sexo femenino de 42 años sin antecedentes clínicos de relevancia, con hipopotasemia severa y cambios electrocardiográficos imitando isquemia miocárdica con enfermedad coronaria de múltiples vasos. 
Electrocardiographic changes associated with hypokalemia: ST-segment abnormalities mimicking ischemia of multiple territories

A 42-year-old woman with no previous medical history developed severe hypokalemia. The ECG changes suggested multivessel coronary artery disease with ischemia in several territories

Key Words: hypokalemia, myocardial ischemia, electrocardiogram 


\section{Introducción:}

Las alteraciones en los niveles de potasio séricos tienen efecto en la conducción de las células cardíacas y pueden llevar a cambios electrocardiográficos ${ }^{1}$. Estos cambios incluyen alteraciones de la onda $\mathrm{T}$ y la onda U. Sin embargo, cambios difusos en el ST han sido descritos y deben ser diferenciados de isquemia subendocárdica difusa ${ }^{2}$.

En muchas situaciones de la práctica clínica diaria, el diagnóstico diferencial con la isquemia miocárdica puede ser difícil, especialmente en casos donde las alteraciones del segmento ST son acompañadas por dolor precordial.

\section{CASO CLINICO}

Mujer de 42 años con antecedentes de hipertensión gestacional y cuadro de gastroenteritis aguda 5 días previos a la consulta. Consulta por palpitaciones, asociadas a náuseas y vómitos con dolor precordial de intensidad 2/10 de carácter punzante, modificado por los cambios de decúbito, sin irradiación, de una hora de duración. Al examen físico presentaba taquicardia de $110 \mathrm{lpm}$, PA 110/60, buena perfusión periférica sin signos de sobrecarga de volumen, soplo protosistólico de intensidad 2/6, mayor en área mitral, que irradia a axila, sin otros hallazgos positivos. El ECG inicial evidenció taquicardia sinusal, con supradesnivel del ST de $2 \mathrm{~mm}$ en
aVR y V1 e infradesnivel del ST difuso en el resto de las derivaciones de hasta $3 \mathrm{~mm}$ (Figura 1), y QT prolongado de $0,56 \mathrm{~ms}$. Dichas alteraciones no cambiaron luego de la administración de nitritos sublinguales. Un ecocardiograma transtorácico mostró función sistólica del ventrículo izquierdo conservada, sin trastornos segmentarios de la motilidad parietal y sin valvulopatías. En el laboratorio de ingreso se evidenció K $2,8 \mathrm{mEq} / 1$, y troponina $\mathrm{T}$ ultrasensible de $3 \mathrm{ng} / \mathrm{L}$ al ingreso y de 4 ng/L 3 hrs después.

Se realizó reposición rápida de $\mathrm{K}$ por vía endovenosa. El ECG seriado de control mostró normalización progresiva del segmento ST y descenso de la frecuencia cardíaca sin la administración de betabloqueantes ni vasodilatadores endovenosos (Figura 2).

\section{Discusión:}

La hipopotasemia es una emergencia médica y si no se resuelve rápidamente puede conducir a un paro cardíaco y respiratorio. Y tal como presenta la paciente, el intervalo QTc prolongado refleja una repolarización cardíaca anormal, que es un predictor de arritmia maligna. Dado que la hipopotasemia no tratada se asocia con una alta morbilidad y mortalidad, es fundamental reconocer $\mathrm{y}$ tratar el trastorno de inmediato ${ }^{3}$.

\section{Figura 1: ECG DE INGRESO}

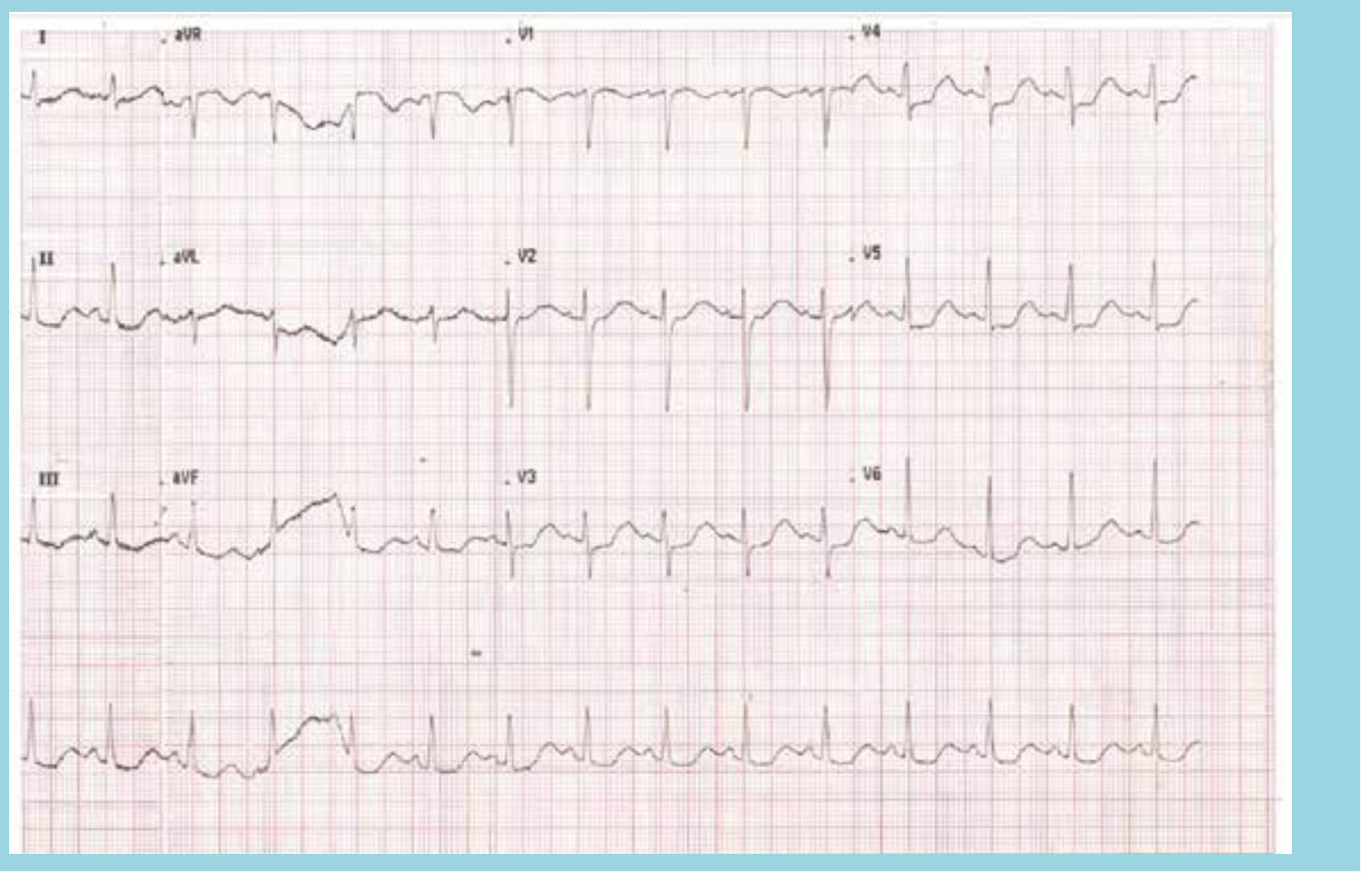



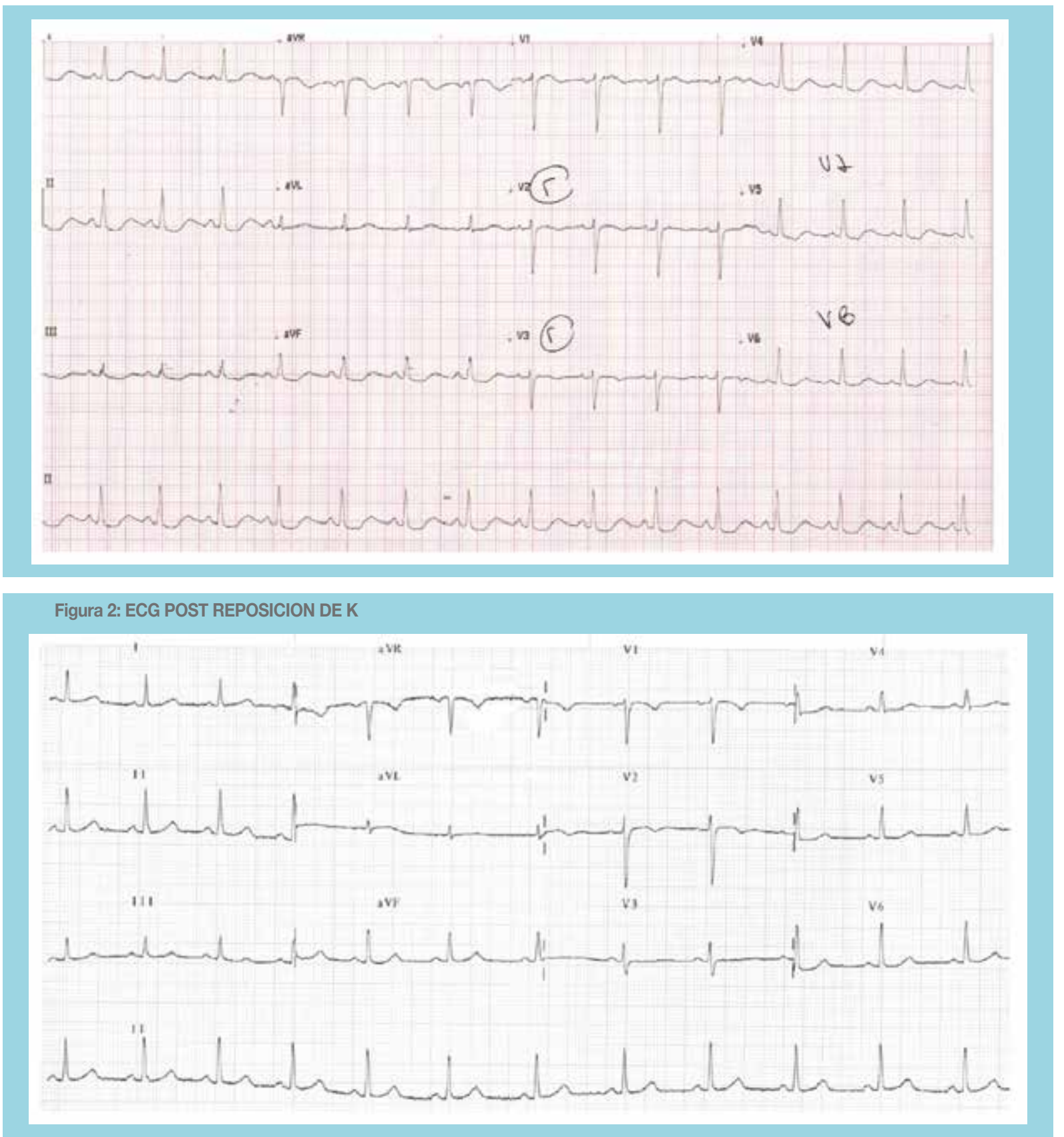

Hay muchas causas, entre ellas la diarrea y el vómito, la reposición de líquidos inadecuada y las drogas, tales como diuréticos tiazídicos y del asa ${ }^{4}$.

El efecto de la hipopotasemia es aumentar el potencial de membrana en reposo, y aumentar la duración del potencial de acción y el periodo refractario, que son cambios potencialmente arritmogénicos. ${ }^{5}$

El diagnóstico electrocardiográfico de hipokalemia no complicada se basa en la depresión del segmento ST, una disminución de la amplitud de la onda $\mathrm{T}$, ondas $\mathrm{U}$ prominentes y una relación onda $\mathrm{U} /$ onda $\mathrm{T}$ mayor que ${ }^{12,7}$. Otras alteraciones descriptas son depresión del segmento $\mathrm{ST}^{2,8,9}$. El patrón de las ondas $\mathrm{U}$ prominentes combinados con segmento ST deprimido y onda T aplanada se conoce como "efecto montaña rusa"7. La depresión del segmento ST requiere una interpretación cuidadosa. Es importante distinguir no isquémico de depresión isquémica ST, que puede no ser una tarea fácil en la práctica clínica ${ }^{6}$. La depresión del segmento ST y la inversión de la onda $\mathrm{T}$ son alteraciones electrocardiográficas comunes. Conocer las diversas características morfológicas isquémicas y no isquémicas es 
fundamental para un diagnóstico oportuno de isquemia miocárdica y alteraciones relacionadas con electrolitos o fármacos ${ }^{2}$.

A diferencia de elevación del segmento ST, la depresión del segmento ST no localiza isquemia ${ }^{11}$. Sin embargo, el alcance y la magnitud de la depresión del segmento ST se correlaciona con el grado y la severidad de la isquemia. De hecho, la depresión del segmento ST en ocho o más derivaciones, junto con elevación del segmento ST en las derivaciones aVR y V1, se asocia con una exactitud de predicción de $75 \%$ de enfermedad de tres vasos o del tronco de la arteria coronaria izquierda $12,13,14$.

Nuestra paciente tenía depresión del segmento ST en derivaciones precordiales y supradesnivel del ST en
aVR, teniendo un gran parecido a las manifestaciones electrocardiográficas en la isquemia miocárdica. Los niveles normales de troponina y ecocardiograma sin trastornos de la motilidad parietal, y la respuesta al suplemento de potasio confirman que las anomalías en el ECG fueron secundarios a hipopotesemia severa.

\section{Conclusión:}

La hipopotasemia es una causa rara, pero bien conocida de la depresión ST con taquicardia ${ }^{10}$. La depresión del segmento ST y supradesnivel en aVR puede imitar la isquemia miocárdica. El diagnóstico diferencial puede ser difícil, sobre todo en los casos en que los cambios ST se acompañen con dolor precordial.

\section{Referencias}

1. DIERCKS DB, SHUMAIK GM, HARRIGAN RA, BRADY WJ, CHAN TC. Electrocardiographic manifestations: electrolyte abnormalities. J Emerg Med. 2004; 27:153-160.

2. HANNA EB, GLANCY DL. ST-segment depression and T-wave inversion: Classification, differential diagnosis, and caveats. Review. Cleveland Clinic Journal Of Medicine. 2011

3. RASTEGAR A, SOLEIMANI M. Hypokalaemia and hyperkalaemia. Postgrad Med J. 2001;77:759-764.

4. WEBSTER A, BRADY W, MORRIS F. Recognising signs of danger: ECG changes resulting from an abnormal serum potassium concentration. Emerg Med J. 2002;19:74-77.

5. HELFANT RH. Hypokalaemia and arrhythmias. Am J Med.1986;80:13-22.

6. PETROV DB. Severe Hypokalemia Masquerading Myocardial Ischemia. Cardiol Res. 2012;3:236-238

7. KHALID A, CLERK A, PATEL M. Severe ST depression due to hypokalemia mimicking ischaemia. J Assoc Physicians India. 2005;53:297

8. GLANCY DL, WANG WL. ECG of the month. Abnormal electrocardiogram in a woman with a urinary tract infection. Sinus rhythm, rate $82 /$ minute. Sagging ST segments, low T waves, and prominent $\mathrm{U}$ waves suggest hypokalemia. J La
State Med Soc 2007; 159:5-7.

9. SURAWICZ B, BRAUN HA, CRUM WB, KEMP RL, WAGNER S, BELLET S. Quantitative analysis of the electrocardiographic pattern of hypopotassemia. Circulation 1957; 16:750-763.

10. SINGH PM, KASHYAP L. Post-operative severe hypokalemia mimicking myocardial ischemia. J Anaesthesiol Clin Pharmacol. 2013; 29(4): 567-568.

11. LI D, LI CY, YONG AC, KILPATRICK D. Source of electrocardiographic ST changes in subendocardial ischemia. Circ Res 1998; 82:957-970.

12. GORGELS AP, VOS MA, MULLENEERS R, DE ZWAAN C, BÄR FW, WELLENS HJ. Value of the electrocardiogram in diagnosing the number of severely narrowed coronary arteries in rest angina pectoris. Am J Cardiol 1993; 72:999-1003.

13. GLANCY DL. Electrocardiographic diagnosis of acute myocardial infarction. J La State Med Soc 2002; 154:66-75.

14. YAMAJI H, IWASAKI K, KUSACHI S, MURAKAMI T, HIRAMI R, HAMAMOTO H, et al. Prediction of acute left main coronary artery obstruction by 12-lead electrocardiography. ST segment elevation in lead aVR with less ST segment elevation in lead V(1). J Am Coll Cardiol 2001; 38:1348-1354. 